\title{
XII INTERNATIONAL LEPROSY CONGRESS, NEW DELHI, INDIA 20-25 FEBRUARY 1984
}

\section{Editorial}

\section{S G BROWNE}

Secretary-General of the Congress, and of the International Leprosy Association

The XII International Leprosy Congress was the best-attended in the long line of such gatherings. It attracted more participants (1460) from more countries (90), and more Abstracts (510) were submitted than at any previous Congress. It is too early to assess its significance in terms of leprosy control, and those who have been bombarded by the plethora of scientific papers over the period of intense activity of concurrent Sessions are perhaps least able to identify the contributions of seminal importance. The Reports of the Workshops, which appear in this issue of Leprosy Review, indicate the range and the depth of topics discussed by correspondence between their members over 2 or 3 years, and the final wording that was thrashed out in the days immediately preceding the Congress.

Official interest in the subject was guaranteed by the personal involvement of the Prime Minister of India, Mrs Indira Gandhi, who readily consented to give the Opening Address - a more-than-adequate compensation for having to postpone the agreed date of the Congress. (Mrs Gandhi called a Consultation of Commonwealth Prime Ministers for the period when the authorities had agreed to allow the Congress the use of the meeting rooms at the Vigyan Bhavan Conference Centre.) The President of India graced the Opening Ceremony with his presence, and Mother Teresa was among the participants.

Having got off to a good start, the Congress settled down to real work on the Monday afternoon. In order to fit in the numbers of excellent Abstracts submitted and selected by the President's Reading Committee to be presented as full papers (284 of them), concurrent Sessions had been organized. This was unfortunately necessary, but it did lead to clashes of interest where participants had to choose one of the two sessions they would like to attend. However, the organizers had striven to reduce these possible clashes to a minimum by a deft arrangement of the sessions. The most noticeable exception to the general rule of concurrent Sessions was on the Friday when there were two sessions on the Social Aspects of Leprosy. The Organizing Committee had apparently bowed to pressure from interested parties who wished to emphasize the overriding importance of the non-medical factors in leprosy control. How Raoul Follereau's 
heart would have rejoiced! It was he who, at the Tokyo Congress in 1958, belaboured the participants for being more interested in the fate of the experimental mouse than in the plight of the human victim of leprosy.

Since all the Abstracts submitted will be published in a future issue of the International Journal of Leprosy, and since that issue will contain the comprehensive Report and Summary of the Sessions that I, as Secretary-General of the Congress, gave to the assembled participants at the Closing Session, readers are referred to that Journal for the details that will not be repeated here.

The presentation by poster of the Abstracts (168) proved more popular than was thought probable. Authors were able to explain their findings whenever they found people interested enough to stop and discuss.

An innovation that was much appreciated was the Teaching Seminars, illustrated by coloured transparencies and diagrams. The commentary was in English, French and Spanish - the official languages of the Congress. The series covered several important aspects of leprosy, and brought generalists and specialists up to date. They proved useful and very popular.

In the following paragraphs, the highlights of the Sessions will be indicated and those contributions likely to prove of general interest.

In the Clinical Session, it was reported that in some good leprosy control programmes, the deformity rate among newly diagnosed cases had shown progressive reduction year by year, an observation that suggests that more patients are being diagnosed at an earlier stage: the implication is that there is generally a greater awareness of the cutaneous manifestations of early leprosy and that the treatment being instituted should prevent nerve damage and its sequelae. Practising doctors and medical auxiliaries should become conversant with the range of locally prevalent dermatoses, so as to be able to diagnose and classify more accurately the patients with leprosy presenting at their clinics. The bearing of pregnancy on the appearing of diagnosable leprosy lesions and on the occurrence of reactions was discussed at some length.

The two Sessions on Treatment concentrated on the growing menace of secondary dapsone resistance, and the popularization of regimens of multidrug therapy advocated both to prevent and to treat drug resistance. It was generally agreed that rifampicin should form an essential component of any such regimen because of its mycobactericidal properties. The real danger of monotherapy was emphasized by the report of leprosy bacilli resistant to both dapsone and rifampicin.

It is not yet known if the multidrug regimens that are being advocated will prove efficacious both in preventing the emergence of resistant strains of bacilli and in dealing with persister organisms, but we do know that if they are not adopted, then the resulting drug resistance will pose an unmanageable problem in the whole world.

The place of clofazimine in these regimens was recognized, as was its acceptability by lighter-skinned Mongoloid patients.

In spite of secondary dapsone resistance having appeared in 25 countries, and 
primary resistance being discovered wherever it is being looked for, dapsone is still an excellent anti-leprotic and should not be lightly discarded.

The Malta Leprosy Eradication Project was referred to, and some doubt was expressed about the hepatotoxicity of its prothionamide component. Thalidomide was reported to be most effective in controlling the skin manifestations and systemic symptoms of some types of acute reaction.

As was to be expected, the (three) Sessions on Immunology provided the most popular and the most important forum of the Congress. Workers in many countries are using new and sophisticated techniques for investigating the complexities of the immune response to leprosy infection. A specific phenolic glycolipid has been isolated and identified. Monoclonal antibodies have been synthesized and examined. The search for a protective vaccine continues, with pride of place being apparently accorded at the moment to a mixture of (live) BCG and various moieties obtained from armadillo-derived Mycobacterium leprae. The organisms are being purified by novel techniques to rid them of the last traces of contaminating armadillo elements. Now that quantities of such bacilli are available, work is proceeding apace on the identification of $M$. leprae-specific determinants that are recognized by $\mathrm{T}$-cells. The details of the immune response are found to be increasingly complex, and the role of macrophages as well as that of sensitized lymphocytes is the subject of much fruitful investigation.

With the application of ever-increasingly severe criteria for the identification of $M$. leprae, no candidates were admitted, though the successful culture of closely related mycobacteria may eventuate in the identification of some organism that has an antigenic constitution closely resembling that of M. leprae. Such an organism could be considered as a possible candidate vaccine if it could be shown to sensitize lymphocytes to recognize and to lyse $M$. leprae.

Much work is proceeding on the characterization of the circulating immune complexes in patients with leprosy reaction.

While much of this new work is of fascinating interest, it is to be hoped that some of it at least will soon be seen to have a bearing on the continuing problem of leprosy control.

The Session on Surgery and Rehabilitation was noteworthy in that the gap between the interventionists and the non-interventionists in the matter of the acutely inflamed peripheral nerve in leprosy is evidently narrowing. If antiinflammatory medical treatment does not lead to rapid improvement (shown by diminutive of local pain and tenderness and swelling), then the nerve sheath should be incised to relieve tension.

Reconstructive surgeons are making significant contributions to neurophysiology as they pursue their researches in nerve damage in leprosy.

Time and again, it was emphasized that if only leprosy were diagnosed early and treated properly, then the great bulk of nerve damage and its unfortunate orthopaedic sequelae would be a thing of the past.

The Session on Opthalmology produced some interesting papers on operative 
procedures and on the ultrastructure of the eye damaged in the course of leprosy. Involvement of both the facial (VII) and the trigeminal (V) nerves in leprosy poses problems when the insensitive cornea is exposed in paralytic lagophthalmos - a challenge to the practising leprologist.

The perennial questions posed by nerve damage in leprosy were brought up during the Session devoted to this theme. The entrapment syndrome was discussed, and the possible reasons for maximum damage at the sites of predilection were examined. Breakdown products of both nerve fibres and $M$. leprae may be involved in the pathogenesis of the damage observed - a condition having parallels with allergic peripheral polyneuritis. One interesting report provided evidence that $M$. lepraemurium could cause damage to peripheral nerves in certain strains of mice. Other workers reported invasion of the central nervous system by $M$. leprae in the heavily infected experimental animal.

In the Session on Experimental leprosy, the armadillo was reported to be a most valuable experimental animal. Colonies established in several countries are now the source of $M$. leprae in considerable quantities. The occurrence of infections of wild armadillos by a $M$. leprae-like organism producing a leprosy-like disease is now well established. Recent investigations suggest that this endemic has been present for some time in the southern States of the USA. The 7-banded armadillo in Argentina has been successfully infected with $M$. leprae. Unlike its relatives further north, this animal breeds in captivity.

Although it has long been a canon of leprosy orthodoxy that the disease is confined to humans, the discovery of leprosy in wild armadillos, and more recently in the sooty Mangabey monkey in Af rica has shattered a long-held belief.

The other interesting finding reported at the Congress was the experimental infection with $M$. leprae of nude mice and athymic Lewis rats.

More work is proceeding on the genetic and lymphocytic control of resistance to subcutaneous inoculation of $M$. leprae, and on animal models of the molecular biology of the host-parasite relation in M. lepraemurium infections in rodents.

The First Session on Epidemiology and Control was introduced by an authoritative and wide-ranging statement from the World Health Organization that emphasized the seriousness of the worldwide endemic of sulphone-resistant leprosy. The only way at present available of containing this threat is the reduction in the réservoir de virus by multidrug therapeutic regimens consistently applied. In some countries, this programme could usefully be combined with the attack on tuberculosis. From China came an excellent report on the success of control measures, and from Vietnam news of an ambitious programme for the eradication of leprosy. An epidemic of leprosy in virgin soil was reported from a Polynesian island, very reminiscent of the explosive epidemic in Nauru half a century ago.

Attempts are still being made to identify nutritional and hormonal environmental factors that may predispose to the development of clinically diagnosable leprosy.

Perhaps the most important paper presented at this Session concerned the 
possibility of leprosy control by means of immunoprophylaxis, using a mixture of BCG and various moieties of $M$. leprae. The enthusiastic advocacy of Venezuelan workers will be tempered by the objective assessment of their results.

Medical auxiliaries need better training in leprosy - this was a message that came over loud and clear. In many leprosy programmes, their diagnosis is more accurate than their attempts at classification; since the duration of treatment recommended depends largely on accurate classification, the standard should be raised and better laboratory cover provided.

The second Session discussed strategies aimed at strengthening case detection activities associated with programmes for Primary Health Care. Subclinical leprosy infection may be much commoner than many imagine, but it is not very important from the epidemiological standpoint.

At this Session, it was reported with some satisfaction that the percentage of newly diagnosed leprosy patients who already have some degree of established deformity is decreasing in areas where good control programmes are in operation.

A potentially useful urine spot test to detect regularity of dapsone intake was described, but it may not be of great practical value in programmes where multidrug therapy is the norm.

Genetic studies in leprosy and the possible linkage of certain histocompatibility antigens (on the HLA system) are helping to resolve the earlier reported discordances. Twin-pair investigations give concordance rates of infection and type of leprosy significantly higher in monozygotic than in dizygotic twins.

Some good papers on the various drugs used in the treatment of leprosy were presented at the Session on Experimental Therapy. An important and practical recommendation concerned the value of a monthly dose of rifampicin as being therapeutically effective and less hepatotoxic than intermittent dose regimens with shorter intervals between doses. One case of resistance to clofazimine has been reported, but quite a number of instances of resistance to rifampicin are now known. It would be a tragedy if the efficacy of this valuable mycobactericidal drug were to be impaired by uncontrolled and indiscriminate use.

To accommodate several presentations that did not fit easily into the announced Sessions, time was allotted to the study of various aspects of the Pathology of leprosy. The importance of the vascular endothelium in the symptomatology of leprosy was emphasized. One paper suggested that visceral lesions are more common than is generally thought. The well-established observation that leprosy bacilli may leave the intact skin through sebaceous and sweat glands and hair follicles was mentioned. It is more difficult to identify the portal of entry, but recent work incriminates the mucosa of the posterior nasal region.

For the first time in the history of Congress held under the auspices of the International Leprosy Association, two whole sessions - with no competitive concurrent Sessions - were devoted to the Social aspects of leprosy. This fact indicates the importance that all leprosy workers now attach to the non-medical, 
non-clinical aspects of leprosy. The epidemiology of leprosy cannot be studied apart from its social and cultural milieu, and apart from all the factors of beliefs and assumptions, of language and superstitions, surrounding the disease. Nor can control measures be successfully implemented unless the co-operation of everybody concerned-patients, families, fellow-villagers, the government and medical personnel-is enlisted.

The Session discussed the pros and cons of substituting the term 'Hansen's disease' for leprosy, but was unconvinced by the arguments put forward. The problems and opportunities presented by the WHO slogan 'Health for all by the year 2000', and the present fashionable insistence on Primary Health Care, were discussed in the context of the differing situations in the countries were leprosy constitutes a disease of public health importance.

The Session identified target groups towards which specific health education programmes should be initially directed-medical staff at leprosy institutions and, indeed, all doctors and medical students; the educated patient; and those patients not suffering from the deformities associated in the public mind with leprosy.

There is a regrettable gap between the new knowledge about leprosy and its treatment, and the application of this knowledge to the individual sufferer. So far, many of the latter are not convinced of the effectiveness of leprosy treatment and its success in preventing deformity.

The hope was expressed that at future Congresses it might be possible to welcome the paticipation of leprosy patients in discussions on the social aspects of leprosy; this would give point and dramatic urgency to the theorizing of the pundits.

As in most congresses nowadays, the Programme for the Sessions was overfull, and there was inadequate time for discussion. Many of the presentations merited immediate examination by groups of interested and knowledgeable critics. The thrust and parry of good debate would add immeasurably to the value of these international gatherings. But the difficulties are immense, almost insuperable. Unless potential participants can ensure that their travel and accommodation costs will be met whether they present papers or not, attendence at future Congresses will suffer; overcrowded programmes do not provide the best environment for unhurried objective examination of important contributions to knowledge.

There is no doubt, however, that the XII International Leprosy Congress will go down in history as the biggest - and perhaps the best - of these gatherings of people from many lands and many scientific and social disciplines engaged in the common task of tackling one of the most redoubtable foes still confronting the human race. 